\title{
Optical properties of metallic (III, Mn)V ferromagnetic semiconductors in the infrared to visible range
}

\author{
E. M. Hankiewicz, ${ }^{1}$ T. Jungwirth, ${ }^{2,3}$ T. Dietl, ${ }^{4,5}$ C. Timm, ${ }^{6}$ and Jairo Sinova ${ }^{1}$ \\ ${ }^{1}$ Department of Physics, Texas A\&M University, College Station, Texas 77843-4242, USA \\ ${ }^{2}$ Institute of Physics, ASCR, Cukrovarnická 10, 16253 Praha 6, Czech Republic \\ ${ }^{3}$ Department of Physics, University of Texas, Austin, Texas 78712-0264, USA \\ ${ }^{4}$ Institute of Physics, Polish Academy of Sciences, al. Lotników 32/46, PL-02-668 Warszawa, Poland \\ ${ }^{5}$ ERATO Semiconductor Spintronics Project, Japan Science and Technology Agency, al. Lotników 32/46, \\ PL-02-668 Warszawa, Poland \\ ${ }^{6}$ Institut für Theoretische Physik, Freie Universität Berlin, Arnimallee 14, D-14195 Berlin, Germany \\ (Received 26 February 2004; revised manuscript received 16 August 2004; published 20 December 2004)
}

\begin{abstract}
We report on a study of the ac conductivity and magneto-optical properties of metallic ferromagnetic (III, $\mathrm{Mn}) \mathrm{V}$ semiconductors in the infrared to visible spectrum at zero temperature. Our analysis is based on the successful kinetic exchange model for (III, Mn)V ferromagnetic semiconductors. We perform the calculations within the Kubo formalism and treat the disorder effects pertubatively within the Born approximation, valid for the metallic regime. We consider an eight-band Kohn-Luttinger model (six valence bands plus two conduction bands) as well as a ten-band model with additional dispersionless bands simulating phenomenologically the upper-mid-gap states induced by antisite and interstitial impurities. These models qualitatively account for optical-absorption experiments and predict new features in the mid-infrared Kerr angle and magnetic-circulardichroism properties as a function of Mn concentration and free carrier density.
\end{abstract}

DOI: 10.1103/PhysRevB.70.245211

PACS number(s): 75.50.Pp, 78.20.Ls

\section{INTRODUCTION}

The desire to integrate storage capabilities and information processing in single semiconductor-based devices has fueled the development of ferromagnetic semiconductors based on (III, Mn)V materials. ${ }^{1-4}$ These materials have been the focus of intensive research over the recent years after nonequilibrium growth procedures ${ }^{5,6}$ have demonstrated the ability to achieve a ferromagnetic phase by increasing the hole-carrier concentration. Holes are introduced by $\mathrm{Mn}$, which acts as an acceptor when substituted for a cation. The improvement in the carrier concentration is due to a decrease in the concentration of compensating defects, such as Asantisites and Mn-interstitials. These defects act as donors and hence reduce the free-carrier concentration with respect to the substitutional-Mn density. The donor defect concentration can be diminished even further by low temperature annealing of these materials. ${ }^{7-12}$ Electron paramagnetic resonance (EPR) and optical measurements ${ }^{13-17}$ show that the $d$-electrons of the Mn impurity are strongly localized with total spin $S=5 / 2$. Also, the neutral impurity state consisting of a $3 d^{5}$ configuration plus a weakly bound hole is only observed experimentally for very low Mn concentrations outside the doping range where ferromagnetism occurs. Therefore, the holes are considered to reside in delocalized or weakly localized valence-band states.

For the doping regime where the highest conductivities are obtained, these experimental considerations lead to a popular model starting from a Hamiltonian of the form

$$
\begin{aligned}
H= & H_{\text {host }}+J_{\mathrm{pd}} \sum_{I, i} \mathbf{S}_{I} \cdot \mathbf{s}_{i, \text { holes }} \delta\left(\mathbf{r}_{i}-\mathbf{R}_{I}\right) \\
& +J_{\text {sd }} \sum_{I, i} \mathbf{S}_{I} \cdot \mathbf{s}_{i, \text { elec }} \delta\left(\mathbf{r}_{i}-\mathbf{R}_{I}\right),
\end{aligned}
$$

where $H_{\text {host }}$ is the Hamiltonian describing the electronic structure of the host semiconductor, which can be limited to a given subset of the bands such as the valence bands (holes), conduction bands (electrons), or both, and the last two terms are the exchange coupling between the localized $d$-electrons and the delocalized holes $(p)$ and electrons $(s) .{ }^{18}$ This effective Hamiltonian incorporates the effects of spin-orbit coupling, which plays a very important role in the qualitative and quantitative understanding of these materials. ${ }^{19,20}$ The properties predicted by this model are most easily understood in the strongly metallic regime for which disorder in the spatial distribution of the $\mathrm{Mn}^{2+}$ ions and other defects of the materials can be treated perturbatively. The model can be further simplified by the mean-field approximation where usually the virtual crystal approximation (VCA) is made. ${ }^{18,20}$ Disorder effects have been taken into account by the introduction of a finite quasiparticle lifetime within the Born approximation. The accurate description of many thermodynamic and transport properties of metallic ( $\mathrm{Ga}, \mathrm{Mn}) \mathrm{As}$ samples, such as the transition temperature, ${ }^{18,19,21}$ the anomalous Hall effect, ${ }^{22,23}$ the anisotropic magnetoresistance, ${ }^{22,24}$ the magneto-crystalline anisotropy, ${ }^{19,25,26}$ the spin-stiffness, ${ }^{26}$ the ferromagnetic domain wall widths, ${ }^{27}$ the magnetic dynamic damping coefficients, ${ }^{28}$ and the magneto-optical properties, ${ }^{19,29,30}$ has proven the merit of this effective 
Hamiltonian approach and the perturbative treatment of disorder within the metallic regime. Disorder effects in diluted magnetic semiconductors, including the case of strong disorder, have recently been reviewed in Ref. 31 .

Optical properties are among the key probes into the electronic structure of materials. Given the wide energy range that optical probes can attain, many different physical effects can be addressed by exploring the full spectrum of phenomena including the visible and infrared absorption, ${ }^{32-38}$ magneto-optical effects such as the magnetic circular dichroism, ${ }^{38-41}$ Raman scattering, ${ }^{42-44}$ photoemission, ${ }^{45-49}$ cyclotron resonance, ${ }^{50-52}$ and ellipsometry measurements. ${ }^{53}$

In earlier work some of us explored the theoretical predictions for the infrared magnetooptical properties of these materials within a six-band model and found them to agree quantitatively with experiments in the strongly metallic regime. ${ }^{29,30,54}$ With better samples and a wider spectrum now available in experiments, there is the need to explore the predictions in this broader range. Hence in this paper we extend our model to the whole range from infrared to optical frequencies. We calculate the infrared and optical conductivity, as well as magneto-optical effects, for the typical level of compensation in samples measured so far $^{32,33,35-37}$ as well as for the more metallic regime of optimally annealed samples, for which our theory is most suitable. We also compute the dependence of magneto-optical effects on compensation and Mn concentration, which should be useful for detailed comparison with experiments, providing further insights in the validity of this model. The strong effects predicted by our calculations should motivate further experimental exploration of the complex magneto-optical effects in these materials beyond the optical regime. We use the $\mathbf{k} \cdot \mathbf{p}$ or KohnLuttinger $(\mathrm{KL})$ model $^{55}$ to describe the carriers, which are coupled with the $S=5 / 2 \mathrm{Mn}$ moments by $p$ - $d$ exchange treated in the mean-field approximation. ${ }^{18}$ We consider an eight-band (six valence plus two conduction bands) KL model as well as a ten-band model with additional dispersionless bands simulating the localized upper mid-gap states induced by the antisite impurities, interstitial impurities, and possibly by intra- $d$-shell transitions within the Mn. ${ }^{19}$

Dynamical-mean-field-theory studies, ${ }^{56}$ which use a single-band model that neglects the spin-orbit coupling and the heavy-light degeneracy of a III-V semiconductor valence band, demonstrate that non-Drude impurity-band-related peaks in the frequency-dependent conductivity occur in DMS ferromagnet models when the strength of the exchange interaction is comparable to the valence-band width. Although these insights are useful, the conductivities predicted by this model are inconsistent with experiment in their temperature dependence and do not, by construction, incorporate strong magneto-optical effects in the mid-infrared regime which we find to be important. In a recent related investigation of the optical properties using Monte Carlo simulations, the transition from the valence band to a Mn impurity band was studied. ${ }^{57}$ However these approaches miss the important multiband structure of (III, Mn)V which seems to be a key to understand the magneto-optical properties of these compounds in the metallic regime and the anisotropies mentioned above.

The paper is organized as follows: In Sec. II we briefly describe our theoretical approach and approximations and present the model Hamiltonian. In Secs. III A and III B we analyze the results for the eight- and ten-band models. We stress the strong dependence between the concentration of carriers and the amplitude and shift of magneto-optical effects. We compare theoretical results with experimental ones emphasizing the role of the localized band in explaining optical experiments. ${ }^{33}$ We summarize our results in Sec. IV.

\section{THEORETICAL APPROACH}

Our theoretical approach starts by coupling the valenceband electrons with $S=5 / 2 \mathrm{Mn}$ local moments with a semiphenomenological local exchange interaction, as shown in Eq. (1). We employ an eight- or ten-band KL Hamiltonian $H_{\mathrm{KL}}$ for the carriers in the host GaAs and treat the Mn local moments within mean-field theory and VCA. ${ }^{18}$ At zero temperature this gives rise to valence and conduction bands splitting by the effective exchange fields $\mathbf{h}_{p d / s d}=N_{\mathrm{Mn}} S J_{p d / s d} \hat{\mathbf{z}}$, where $N_{\text {Mn }}$ is the substitutional Mn density and the strength of the exchange coupling between the valence $(p)$ and conduction $(s)$ electrons is taken to be $J_{p d}=55 \mathrm{meV} \mathrm{nm}^{-3}$ and $J_{s d}=-9 \mathrm{meV} \mathrm{nm}^{-3}$, respectively, as obtained from photoemission, resistance, and magnetic-circular-dichroism (MCD) measurements. ${ }^{45,58,59}$ We assume that the magnetization is aligned along the growth $(\hat{\mathbf{z}})$ direction by a small external magnetic field. We also restrict ourselves to the $T$ $=0$ limit, which allows us to neglect scattering off thermal fluctuations in the orientation of Mn moments. We assume collinear magnetization in the ground state, ignoring the possibility of disorder-induced noncollinearity in the ground state which is known to be less likely for the strongly metallic (III, Mn)V ferromagnets which we focus on. ${ }^{60-62}$

The ac-conductivity tensor is calculated within the Kubo formalism described in earlier studies. ${ }^{29,30}$ The sources of disorder known to be relevant in these materials include positional randomness of the substitutional $\mathrm{Mn}$ ions with charge $Q=-e$ and random placement of interstitial Mn ions and As antisites, both acting as non-magnetic double donors with charge $Q=+2 e .^{63}$ Previous estimates of the valenceband quasiparticle lifetimes using Fermi's Golden Rule including screened Coulomb and exchange interactions and the effect of compensation are of the order of $100 \mathrm{meV} .{ }^{24} \mathrm{We}$ introduce this disorder effect within the Born approximation in the Kubo formalism as in Refs. 29 and 30

In the calculations the Mn concentration $x$ and the freecarrier concentration $p$ are treated as independent parameters. Compensation of the holes introduced by the Mn acceptors is due to antisite and interstitial defects, as noted above. Recently developed annealing procedures allow some independent experimental control over $x$ and $p .^{2,8-12,64}$

Model Hamiltonians: In the VCA, the interactions are replaced by their spatial averages, so that the Coulomb interaction vanishes and holes interact with a homogeneous exchange field. The unperturbed Hamiltonian for the valence and conduction bands reads $H_{0}=H_{\mathrm{KL}}+\mathbf{h} \cdot \mathbf{s}$, where $H_{\mathrm{KL}}$ is the eight-band KL Hamiltonian of pure GaAs, and $\mathbf{h}$ is the exchange field that splits the valence and conduction bands, 
respectively, as mentioned in the previous section. To take into account the considerable contribution from donor defect-induced states below the conduction-band edge, we phenomenologically add two dispersionless bands. We assume that the latter are composed of localized states of mainly $s$-type. Choosing the angular momentum quantization direction along the $z$-axis, the basis functions corresponding to heavy, light, split-off holes, conduction-band electrons, and defect bands can be written, in this order, as

$$
\begin{gathered}
|1\rangle=\frac{-1}{\sqrt{2}}\left(X_{\uparrow}+i Y_{\uparrow}\right), \\
|2\rangle=\frac{1}{\sqrt{6}}\left(X_{\uparrow}-i Y_{\uparrow}\right)+\sqrt{\frac{2}{3}} Z_{\downarrow}, \\
|3\rangle=\frac{-1}{\sqrt{6}}\left(X_{\downarrow}+i Y_{\downarrow}\right)+\sqrt{\frac{2}{3}} Z_{\uparrow},
\end{gathered}
$$

$$
\begin{gathered}
|4\rangle=\frac{1}{\sqrt{2}}\left(X_{\downarrow}-i Y_{\downarrow}\right), \\
|5\rangle=\frac{-1}{\sqrt{3}}\left(X_{\downarrow}+i Y_{\downarrow}\right)-\sqrt{\frac{1}{3}} Z_{\uparrow}, \\
|6\rangle=\frac{-1}{\sqrt{3}}\left(X_{\uparrow}-i Y_{\uparrow}\right)+\sqrt{\frac{1}{3}} Z_{\downarrow}, \\
|7\rangle=S_{\uparrow}, \\
|8\rangle=S_{\downarrow}, \\
|9\rangle=S_{\uparrow}^{\text {an }}, \\
|10\rangle=S_{\downarrow}^{\text {an }},
\end{gathered}
$$

where $X, Y, Z$ are $p$-like orbitals, $S$ and $S_{a n}$ are the $s$ orbitals associated with conduction and defect bands, respectively. In this basis, the ten-band KL Hamiltonian has a form

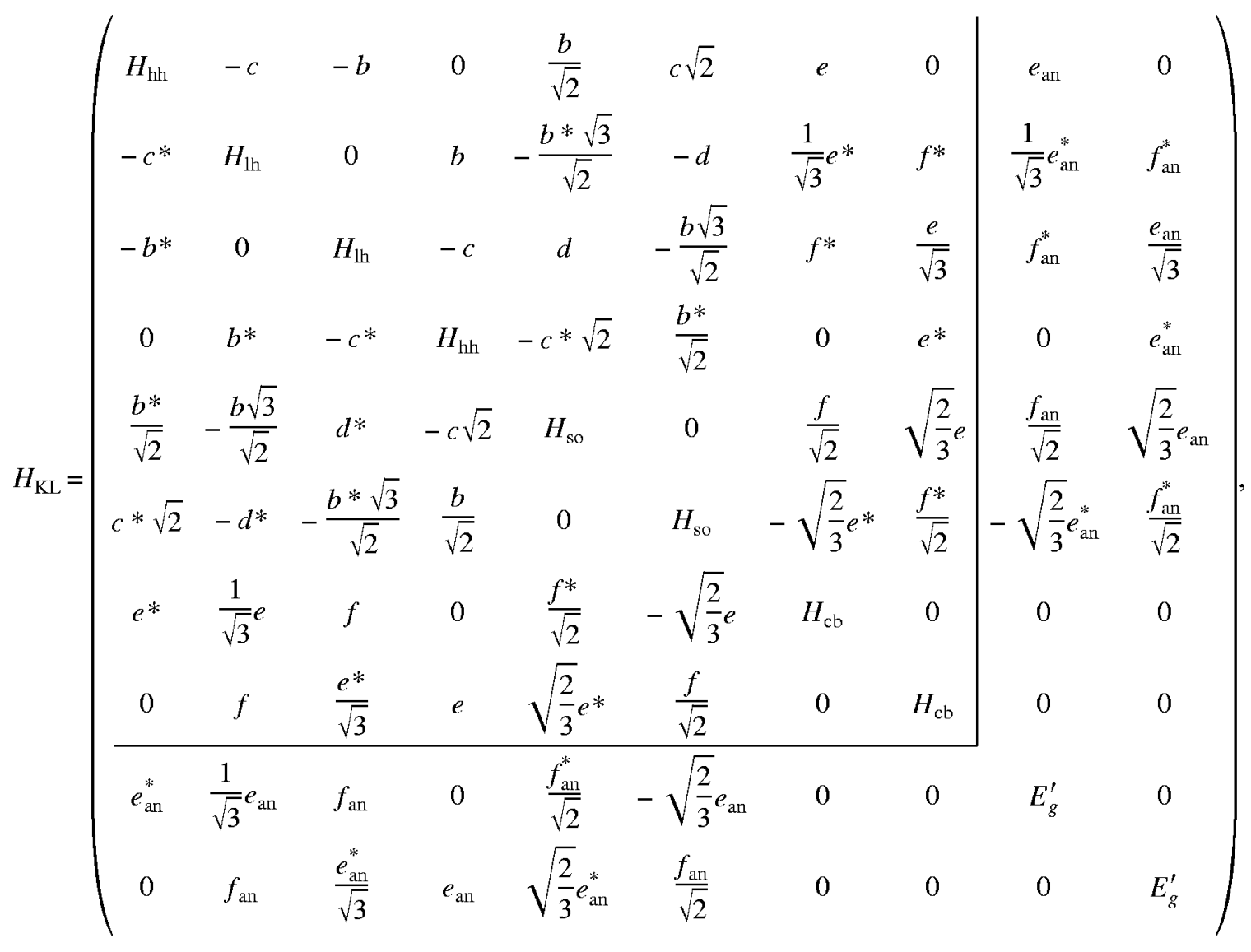




$$
H_{\mathrm{KL}}=\left(\begin{array}{cccccccccc}
H_{\mathrm{hh}} & -c & -b & 0 & \frac{b}{\sqrt{2}} & c \sqrt{2} & e & 0 & e_{\mathrm{an}} & 0 \\
-c^{*} & H_{\mathrm{lh}} & 0 & b & -\frac{b^{*} \sqrt{3}}{\sqrt{2}} & -d & \frac{1}{\sqrt{3}} e^{*} & f^{*} & \frac{1}{\sqrt{3}} e_{\mathrm{an}}^{*} & f_{\mathrm{an}}^{*} \\
-b^{*} & 0 & H_{\mathrm{lh}} & -c & d & -\frac{b \sqrt{3}}{\sqrt{2}} & f^{*} & \frac{e}{\sqrt{3}} & f_{\mathrm{an}}^{*} & \frac{e_{\mathrm{an}}}{\sqrt{3}} \\
0 & b^{*} & -c^{*} & H_{\mathrm{hh}} & -c^{*} \sqrt{2} & \frac{b^{*}}{\sqrt{2}} & 0 & e^{*} & 0 & e_{\mathrm{an}}^{*} \\
b^{*} & -\frac{b \sqrt{3}}{\sqrt{2}} & d^{*} & -c \sqrt{2} & H_{\mathrm{so}} & 0 & \frac{f}{\sqrt{2}} & \sqrt{\frac{2}{3}} e & \frac{f_{\mathrm{an}}}{\sqrt{2}} & \sqrt{\frac{2}{3}} e_{\mathrm{an}} \\
c^{*} & -d^{*} & -\frac{b^{*} \sqrt{3}}{\sqrt{2}} & \frac{b}{\sqrt{2}} & 0 & H_{\mathrm{so}} & -\sqrt{\frac{2}{3}} e^{*} & \frac{f^{*}}{\sqrt{2}} & -\sqrt{\frac{2}{3}} e_{\mathrm{an}}^{*} & \frac{f_{\mathrm{an}}^{*}}{\sqrt{2}} \\
e^{*} & \frac{1}{\sqrt{3} e} & f & 0 & \frac{f^{*}}{\sqrt{2}} & -\sqrt{\frac{2}{3}} e & H_{\mathrm{cb}} & 0 & 0 & 0 \\
0 & f & \frac{e^{*}}{\sqrt{3}} & e & \sqrt{\frac{2}{3}} e^{*} & \frac{f}{\sqrt{2}} & 0 & H_{\mathrm{cb}} & 0 & 0 \\
e_{\mathrm{an}}^{*} & \frac{1}{\sqrt{3}} e_{\mathrm{an}} & f_{\mathrm{an}} & 0 & \frac{f_{\mathrm{an}}^{*}}{\sqrt{2}} & -\sqrt{\frac{2}{3}} e_{\mathrm{an}} & 0 & 0 & E_{g}^{\prime} & 0 \\
0 & f_{\mathrm{an}} & \frac{e_{\mathrm{an}}}{\sqrt{3}} & e_{\mathrm{an}} & \sqrt{\frac{2}{3}} e_{\mathrm{an}}^{*} & \frac{f_{\mathrm{an}}}{\sqrt{2}} & 0 & 0 & 0 & E_{g}^{\prime}
\end{array}\right),
$$

where the eight-band Hamiltonian is the highlighted sector. The quantities that appear in $H_{\mathrm{KL}}$ are:

$$
\begin{gathered}
H_{c b}=E_{g}+\frac{\hbar^{2}}{2 m_{c}^{*}}\left(k_{x}^{2}+k_{y}^{2}+k_{z}^{2}\right) ; \\
H_{h h}=-\frac{\hbar^{2}}{2 m_{0}}\left[( \gamma _ { 1 } + \gamma _ { 2 } - \frac { E _ { \mathrm { an } } } { 2 ( E _ { g } ^ { \prime } + \Delta _ { \mathrm { so } } / 3 ) } - \frac { E _ { P } } { 2 ( E _ { g } + \Delta _ { \mathrm { so } } / 3 ) } ) \left(k_{x}^{2}\right.\right. \\
\left.\left.+k_{y}^{2}\right)+\left(\gamma_{1}-2 \gamma_{2}\right) k_{z}^{2}\right] ; \\
H_{l h}=-\frac{\hbar^{2}}{2 m_{0}}\left[( \gamma _ { 1 } - \gamma _ { 2 } - \frac { E _ { \mathrm { an } } } { 6 ( E _ { g } ^ { \prime } + \Delta _ { \mathrm { so } } / 3 ) } - \frac { E _ { P } } { 6 ( E _ { g } + \Delta _ { \mathrm { so } } / 3 ) } ) \left(k_{x}^{2}\right.\right. \\
\left.\left.+k_{y}^{2}\right)+\left(\gamma_{1}+2 \gamma_{2}-\frac{E_{\mathrm{an}}}{3\left(E_{g}^{\prime}+\Delta_{\mathrm{so}} / 3\right)}-\frac{E_{P}}{3\left(E_{g}+\Delta_{\mathrm{so}} / 3\right)}\right) k_{z}^{2}\right] ; \\
H_{\mathrm{so}}=-\Delta_{\mathrm{so}}-\frac{\hbar^{2}}{2 m_{0}}\left(\gamma_{1}-\frac{E_{\mathrm{an}}}{3\left(E_{g}^{\prime}+\Delta_{\mathrm{so}} / 3\right)}-\frac{E_{P}}{3\left(E_{g}+\Delta_{\mathrm{so}} / 3\right)}\right)\left(k_{x}^{2}\right. \\
\left.+k_{y}^{2}+k_{z}^{2}\right) ;
\end{gathered}
$$

$$
\begin{aligned}
b= & -\frac{\hbar^{2}}{2 m_{0}} \sqrt{12}\left(\gamma_{3}-\frac{E_{\mathrm{an}}}{6\left(E_{g}^{\prime}+\Delta_{\mathrm{so}} / 3\right)}-\frac{E_{P}}{6\left(E_{g}+\Delta_{\mathrm{so}} / 3\right)}\right) k_{z}\left(k_{x}\right. \\
& \left.-i k_{y}\right) ; \\
c= & -\frac{\hbar^{2}}{2 m_{0}} \sqrt{3}\left[\left(\gamma_{2}-\frac{E_{\mathrm{an}}}{6\left(E_{g}^{\prime}+\Delta_{\mathrm{so}} / 3\right)}-\frac{E_{P}}{6\left(E_{g}+\Delta_{\mathrm{so}} / 3\right)}\right)\left(k_{x}^{2}-k_{y}^{2}\right)\right. \\
- & \left.2 i\left(\gamma_{3}-\frac{E_{\mathrm{an}}}{6\left(E_{g}^{\prime}+\Delta_{\mathrm{so}} / 3\right)}-\frac{E_{P}}{6\left(E_{g}+\Delta_{\mathrm{so}} / 3\right)}\right) k_{x} k_{y}\right] ; \\
d= & -\frac{\hbar^{2}}{2 m_{0}} \sqrt{2}\left[-\left(2 \gamma_{2}-\frac{E_{\mathrm{an}}}{3\left(E_{g}^{\prime}+\Delta_{\mathrm{so}} / 3\right)}-\frac{E_{P}}{3\left(E_{g}+\Delta_{\mathrm{so}} / 3\right)}\right) k_{z}^{2}\right. \\
& \left.+\left(\gamma_{2}-\frac{E_{\mathrm{an}}}{6\left(E_{g}^{\prime}+\Delta_{\mathrm{so}} / 3\right)}-\frac{E_{P}}{6\left(E_{g}+\Delta_{\mathrm{so}} / 3\right)}\right)\right]\left(k_{x}^{2}+k_{y}^{2}\right) ; \\
e & =\frac{i P}{\sqrt{2}}\left(k_{x}-i k_{y}\right) ; \quad f=\sqrt{\frac{2}{3}} i P k_{z} ; \\
e_{\mathrm{an}} & =\frac{i P_{\mathrm{an}}}{\sqrt{2}}\left(k_{x}-i k_{y}\right) ; \quad f_{\mathrm{an}}=\sqrt{\frac{2}{3}} i P_{\mathrm{an}} k_{z} ;
\end{aligned}
$$

where $E_{g}$ is the energy gap between valence and conducting bands, $E_{g}^{\prime}$ is the energy of the dispersionless defect band, $m_{0}$ is the bare electron mass, $m_{c}^{*}$ is the effective mass of conduc- 
tion electrons, for GaAs $m_{c}^{*}=0.067 m_{0}$, and $\Delta_{\text {so }}$ is the energy of spin-orbit splitting. $P$ is the momentum matrix element between conducting and valence bands given by,

$$
P=-\frac{i \hbar}{m_{0}}\left\langle S\left|\hat{p}_{x}\right| X\right\rangle
$$

We introduce the dipole energy $E_{P}=2 m_{0} P^{2} / \hbar^{2}$, and set $E_{P}$ $=22.5 \mathrm{eV}$ in accordance with Ref. 65. Similarly, $P_{\text {an }}$ is the momentum matrix elements between defect level and valence bands defined as

$$
P_{\mathrm{an}}=-\frac{i \hbar}{m_{0}}\left\langle S_{\mathrm{an}}\left|\hat{p}_{x}\right| X\right\rangle .
$$

The corresponding energy associated with the defect level is $E_{\text {an }}=2 m_{0} P_{\text {an }}^{2} / \hbar^{2}$. This energy is a parameter of our model. We assume that $E_{\text {an }}$ is of the order of few $\mathrm{eV}$.

In the six-band KL model the valence-band structure is parametrized by the Luttinger parameters $\gamma_{1}, \gamma_{2}, \gamma_{3}$, and $\Delta_{\mathrm{so}}$. For GaAs the values are $\gamma_{1}=6.98, \gamma_{2}=2.06, \gamma_{3}=2.93$, and $\Delta_{\mathrm{so}}=341 \mathrm{meV}$. For the eight- and ten-bands KL model the corrections from the conduction and defect bands must be consistently taken into account in order to conserve spectral weight.

It has been shown that the energy gap in heavily doped GaAs strongly depends on the carrier concentration. ${ }^{66}$ There are several effects which can cause the gap to be reduced, like band-gap narrowing by many-body effects as well as the band-tailing. ${ }^{66,67}$

The energy gap $E_{g}$ is estimated in accordance with Ref. 66 by the formula: $E_{g}(\mathrm{eV})=1.52-1.6 \cdot 10^{-8}\left[p\left(\mathrm{~cm}^{-3}\right)\right]^{1 / 3}$. For simplicity, the disorder-induced broadening, or quasiparticle lifetime, is taken to be a constant of the order of $100 \mathrm{meV}$ in accordance with earlier estimates. ${ }^{24}$

\section{RESULTS AND DISCUSSION}

\section{A. Eight-band model}

Since the infrared conductivity was investigated in detail in the framework of the six-band model, ${ }^{29}$ we first compare the results of the two models in the expanded range.

In Fig. 1 the real part of the diagonal conductivity $\sigma_{x x}(\omega)$ is plotted for the six- and eight-band models as a function of frequency for a Mn concentration of 5\%, a hole concentration of $p=0.11 \mathrm{~nm}^{-3}$, and a lifetime broadening of $\Gamma$ $=100 \mathrm{meV}$. As discussed earlier, ${ }^{29}$ the features at low frequencies together with the shoulder around $220 \mathrm{meV}$ correspond to the Drude peak combined with transitions between heavy- and light-hole bands. The transition from the heavyhole to the split-off band around $500 \mathrm{meV}$ and from the light-hole to the split-off band around $900 \mathrm{meV}$ are strongly broadened and therefore unobservable. ${ }^{29,54}$ For frequencies above $500 \mathrm{meV}$ one can see the loss of spectral weight at high infrared frequencies in the eight-band model in comparison to the six-band model, which is not considered reliable in this high-frequency range. Of course the spectral weight is not lost but shifted to higher photon energies above $1.5 \mathrm{eV}$ as expected from the $f$-sum rule where total integrated conductivity should remain constant. We identify the

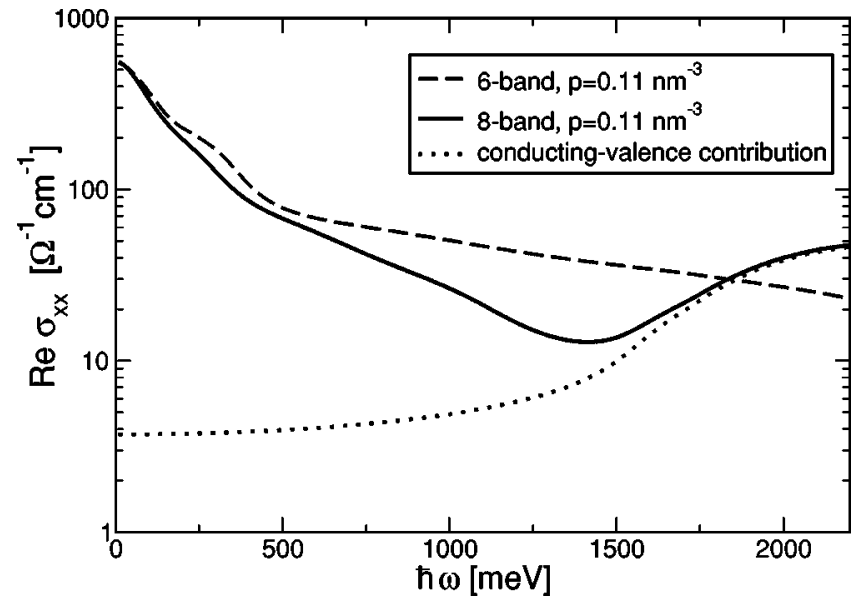

FIG. 1. Real part of the diagonal conductivity, $\operatorname{Re} \sigma_{x x}$, as a function of frequency $\hbar \omega$. The dashed and solid lines correspond to the total $\operatorname{Re} \sigma_{x x}$ for the six- and eight-band models, respectively. The dotted line shows $\operatorname{Re} \sigma_{x x}$ for only valence-conduction-band transitions within the eight-band model. The Mn concentration is $5 \%$, the hole concentration is $p=0.11 \mathrm{~nm}^{-3}$, and the lifetime broadening is $\Gamma=100 \mathrm{meV}$.

upturn around $1.5 \mathrm{eV}$ in the eight-band model as the valenceconduction-band transition (see dotted line in Fig. 1).

In Fig. 2 we show the absorption as a function of frequency for clean and disordered systems with different concentrations of $\mathrm{Mn}$. As a check we first consider a low concentration of $\mathrm{Mn}, x=0.5 \%$. In the clean limit we observe the expected sharp band edge at optical frequencies. When including the disorder effect perturbatively the band edge gets broadened but still identifiable within a few percent of the the result for the clean calculation. For the typical concentration $x=5 \%$ for ferromagnetic $(\mathrm{Ga}, \mathrm{Mn})$ As the absorption is stronger and the band edge also broadened. Note also that due to the $f$-sum rule the spectral weight associated with inter- and intra-valence-band transitions is shifted to higher energies in the disordered case, as observed experimentally.

From the point of view of integration of $(\mathrm{Ga}, \mathrm{Mn}) \mathrm{As}$ in magnetorecording devices the strength of magneto-optical

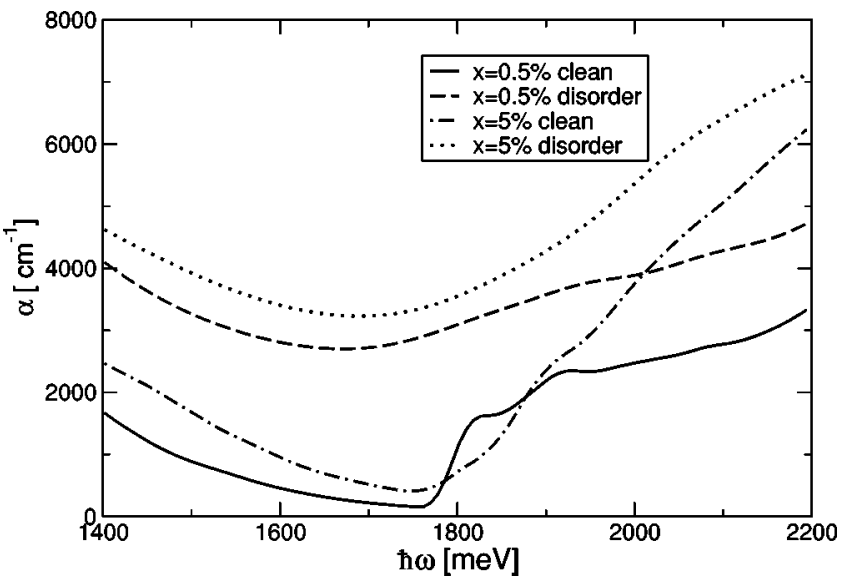

FIG. 2. Absorption as a function of photon energy for Mn concentrations $x=0.5 \%$ and $x=5 \%$ and $p=0.35 \mathrm{~nm}^{-3}$ for a clean system and for a system with disorder induced by impurity scattering. 


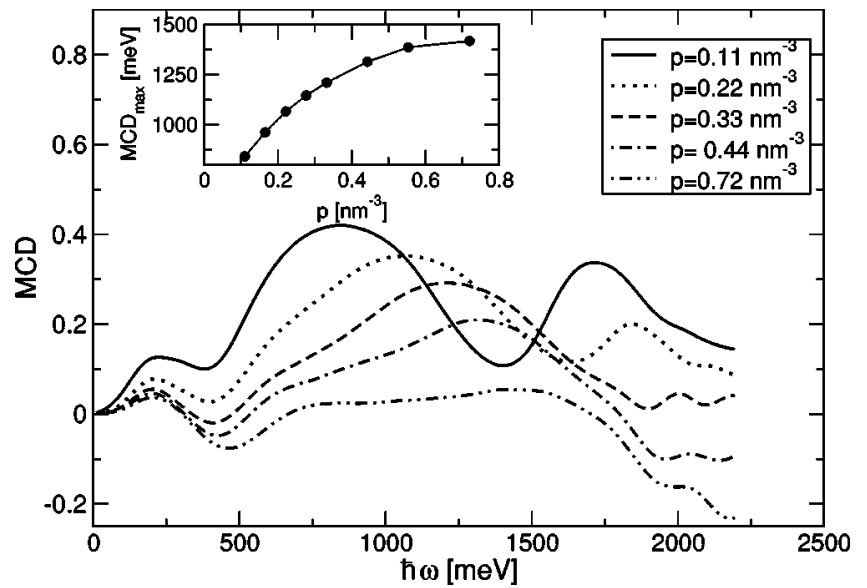

FIG. 3. Magnetic circular dichroism (MCD) as a function of photon energy for different concentrations of holes for $\mathrm{Ga}_{0.95} \mathrm{Mn}_{0.05}$ As. The inset shows the position of the maximum of the MCD spectrum vs hole concentration. The lifetime broadening is $\Gamma=100 \mathrm{meV}$.

effects is very important. ${ }^{69}$ Therefore, we now present our predictions for magnetic circular dichroism (MCD) as well as the Kerr effect as a function of hole concentration. We consider the system air/(Ga, Mn)As/GaAs, taking into account multiple reflections in the thin-film limit of $(\mathrm{Ga}$, Mn)As, i.e., for wavelengths larger than the epilayer thickness. We postpone the discussion of magneto-optical effects in thicker films of $(\mathrm{Ga}, \mathrm{Mn})$ As to the next subsection in the context of the ten-band model.

The MCD signal is defined as the difference of absorption for left $\left(\sigma^{-}\right)$and right $\left(\sigma^{+}\right)$-circularly-polarized light renormalized by the total absorption. ${ }^{68}$ In the thin-film geometry one obtains for the MCD signal

$$
\mathrm{MCD} \equiv \frac{\alpha^{-}-\alpha^{+}}{\alpha^{+}+\alpha^{-}}=\frac{\operatorname{Im} \sigma_{x y}(\omega)}{\operatorname{Re} \sigma_{x x}(\omega)} .
$$

In Fig. 3 we show the MCD signal as a function of frequency for different concentrations of carriers. The strong $p$ - $d$ exchange in diluted magnetic semiconductors causes the splitting of bands. However, it is the strong spin-orbit coupling present in these materials that gives rise to a strong MCD signal in the mid-infrared range. This spin-orbit coupling and the exchange field allow transitions between spin-orbitcoupled bands which give rise to a high $\sigma_{x y}(\omega)$ in the midinfrared regime. The natural energy scales of the Fermi energy and the valence-band splittings are in the infrared range and thus the difference in absorption of differently polarized light should be particularly pronounced in this range as is evident from the theoretical curves. The maximum for small photon energies around $220 \mathrm{meV}$ corresponds to heavy-hole to light-hole transitions while the main maximum in the $800-1400 \mathrm{meV}$ of transitions between spin-orbit split-off holes and heavy/light holes and between valence bands and the conduction band. The frequency of the main maximum is shown in the inset as a function of hole concentration. Its position monotonically increases with carrier concentration. This dependence of the MCD signal could be useful for the

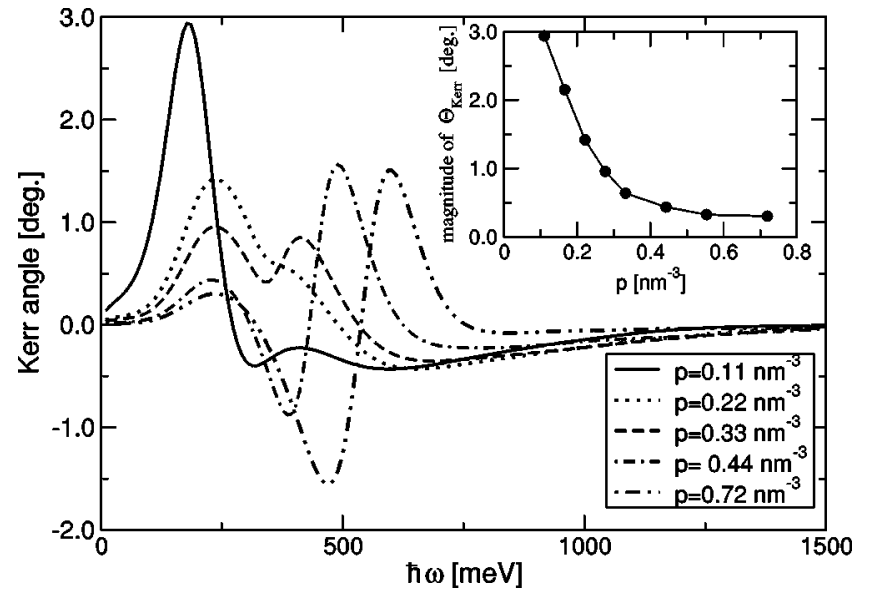

FIG. 4. Kerr angle $\Theta_{\text {Kerr }}$ as a function of frequency for different concentrations of holes for $\mathrm{Ga}_{0.95} \mathrm{Mn}_{0.05} \mathrm{As}$. In the inset we plot the amplitude of the Kerr angle at $\hbar \omega=220 \mathrm{meV}$ as a function of hole concentration. The lifetime broadening is $\Gamma=100 \mathrm{meV}$.

estimation of carrier concentration in experimentally measured (Ga,Mn)As thin films and should be a straightforward test of the validity of this theory.

The experimental MCD spectra of ferromagnetic (Ga,Mn)As semiconductors ${ }^{40,41}$ confirm that $p$ - $d$ exchange interaction is antiferromagnetic in this compound. In the ferromagnetic state this implies a positive MCD and our calculations are in agreement with this picture since we start from a phenomenological effective Hamiltonian model which antiferromagnetically couples the band delocalized quasiparticles and the localized $d$-electron moment. In this sense, the agreement in the optical regime is built in the theory while the spectra calculated in the infrared regime are full nontrivial predictions which should be a much more stringent test of this simple theory.

We next turn to the calculation of the Kerr effect, which occurs in reflection from a magnetic medium. In the thin-film geometry the Kerr angle $\Theta_{\text {Kerr }}$ and the ellipticity $\eta_{\text {Kerr }}$ are defined as ${ }^{69}$

$$
\Theta_{\mathrm{Kerr}}+i \eta_{\mathrm{Kerr}}=\frac{r_{+}-r_{-}}{r_{+}+r_{-}}
$$

where $r_{ \pm}$are the total complex reflection amplitudes for leftand right-circularly-polarized light. The Kerr angle is shown in Fig. 4 as a function of the photon energy for different carrier concentrations and a Mn concentration of $x=5 \%$. The Kerr angle is of the order of a few degrees, comparable to the Kerr effect in materials used in magnetorecording devices. ${ }^{70}$

In the case of the thick layer the Kerr angle is proportional to the imaginary part of the off diagonal conductivity. Interestingly, we found that numerator as well as denominator of Eq. (15) are important contributions to Kerr angle in a case of thin-film. However the numerator which includes the off-diagonal conductivity is responsible for the main features observed in Fig. 4.

In the inset, the value of the Kerr angle at $220 \mathrm{meV}$ is plotted as a function of hole concentration. In contrast to the MCD signal one observes a decrease of the Kerr angle 
around $220 \mathrm{meV}$ with increasing hole concentration. The sharp changes of sign are again associated with different transitions within the valence subbands and the strong spinorbit coupling.

\section{B. Ten-band model}

From earlier studies on low-temperature GaAs (LT-GaAs) it is known that defects affect the electrical and optical properties since they interact with carriers by acting as traps or scattering and recombination centers. ${ }^{66,71-75}$ Experiments revealed the presence of arsenic antisites, arsenic interstitials, and their complexes. ${ }^{76}$ Usually a Coulomb potential is introduced to describe effects of these charged defects. The early papers studied the dependence of the energy gap on the concentration of holes in heavily doped GaAs, using a one-band model and a screened impurity Coulomb potential. ${ }^{66}$ EPR measurements in GaAs showed the formation of two antisites levels $0.52 \mathrm{eV}$ and $0.75 \mathrm{eV}$ above the valence band. ${ }^{71}$ However, the interpretation of the MCD spectra has been controversial until recently: According to Meyer et al. ${ }^{72}$ MCD spectra arise from intracenter transitions from the $A_{1}$ $s$-type ground state of the defect to two $T_{2}$ excited states with $p$-character. On the other hand, the model proposed by Kaufmann and Windscheif ${ }^{73}$ postulates that MCD is a result of transitions between the $s$-state $A_{1}$ of the defect and the valence bands. Recent studies of arsenic antisites in MCD support the second model. ${ }^{74}$ The intensity of the MCD is very weak in undoped GaAs, of the order of $5 \cdot 10^{-3}$.

The situation becomes more complicated if GaAs is doped with Mn. During low-temperature growth under arsenic overpressure the high concentration of Mn leads to the formation of antisites and $\mathrm{Mn}$ interstitials, according to $a b$ initio calculations. ${ }^{77,78}$ Interstitials have been observed in channeling Rutherford backscattering experiments by $\mathrm{Yu}$ et al. ${ }^{10}$ For a total manganese concentration of $x \approx 7 \%$ about $17 \%$ of the manganese impurities were found in interstitial positions. ${ }^{10}$ Theoretical studies predict that interstitials form a localized level $0.9 \mathrm{eV}$ above the valence band. ${ }^{77}$ As an additional complication, a correlated spatial distribution of defects is expected to develop during growth or annealing due to the strong Coulomb interactions between the defects. ${ }^{31,79,80}$ Singley et al $^{33}$ emphasize that the observed band-tail signal near $0.7 E_{g}$ is likely associated with defectinduced upper-mid-gap states as we consider here. However, recent experiments ${ }^{81-84}$ suggest that another candidate may be intra- $d$-shell transitions within the Mn as predicted earlier by in Ref. 19. Hence, without specifying the nature of the mid-gap state we introduce a phenomenological dispersionless band in the gap near $0.7 E_{g}$ to model the system.

In Fig. 5 we show the real part of the diagonal conductivity, $\operatorname{Re} \sigma_{x x}$, as a function of frequency for the ten-band model with $\mathrm{Mn}$ concentration $x=5.2 \%$ and $x=6.66 \%$ and carrier concentration $p=0.345 \mathrm{~nm}^{-3}$ and $p=0.368 \mathrm{~nm}^{-3}$, respectively, and a quasiparticle lifetime of $100 \mathrm{meV}$. Experimental data by Singley et al..$^{33}$ and theoretical fits in the framework of the eight- and ten-band models are shown. The intravalence-band contributions are not included in the spectra shown because the low-frequency conductivity is very

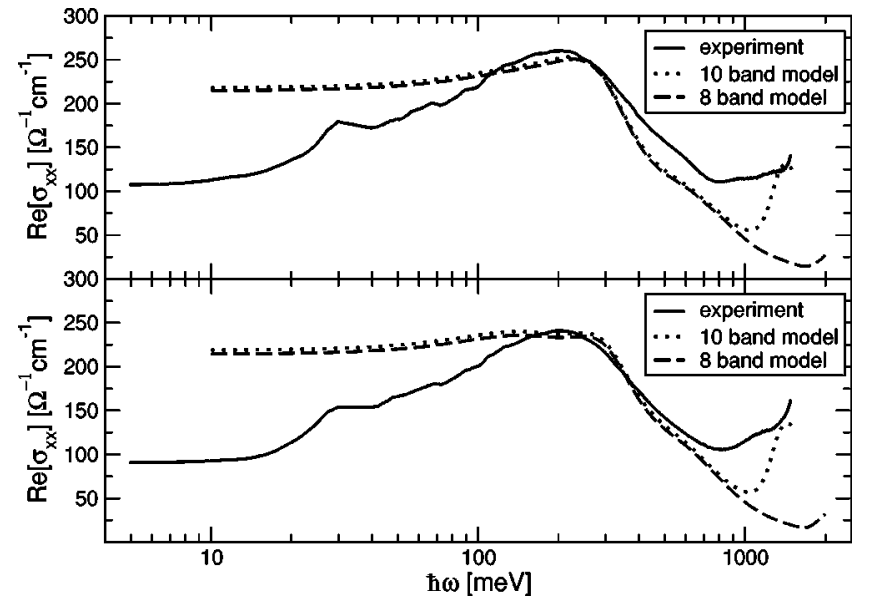

FIG. 5. Real part of the diagonal conductivity, $\operatorname{Re}\left[\sigma_{x x}(\omega)\right]$, as a function of frequency for (a) $x=5.2 \%$ and (b) $x=6.66 \%$. Here we compare experimental results (solid line) with theoretical model for eight-bands (dotted line) and ten-bands (dashed line) model calculate it on. The dispersionless defect energy level, $E_{g}^{\prime}$, is $1 \mathrm{eV}$ above the valence band.

small for these samples, of the order of $100 \Omega^{-1} \mathrm{~cm}^{-1}$. Exact diagonalization studies for these models show a strong suppression of the Drude peak (intraband contribution) for "weakly" metallic samples due to partial localization which cannot be captured by our model and hence its suppression from the spectra shown in Fig. $5 .{ }^{54}$ From this theoretical comparison we estimate a concentration of carriers of $p$ $=0.35 \mathrm{~nm}^{-3}$ and $p=0.37 \mathrm{~nm}^{-3}$ for Mn concentration of $5.2 \%$ and $6.66 \%$, respectively, for the samples measured by Singley et al. ${ }^{33}$ Moreover we find on the basis of similar fits that the compensation in as-grown samples is around $70 \%-80 \%$.

The peak around $220 \mathrm{meV}$ in optical absorption experiments and theoretical data is associated with the intervalence-subband transitions. One can see that the experimental and theoretical positions of this transition are in agreement. ${ }^{32,33}$ We can identify the transitions between lighthole band and split-off band around $500 \mathrm{meV}$ in both experimental and theoretical data. For frequencies larger than $700 \mathrm{meV}$ the eight-band result does not match the experimental data in the upturn observed experimentally around $1.5 \mathrm{eV}$. Note that within the ten-band model the upturn at high energies of the order of $1.5 \mathrm{eV}$ is reproduced. The experimental EPR data for undoped GaAs show that antisite levels lie $0.52 \mathrm{eV}$ and $0.75 \mathrm{eV}$ above the valence band. ${ }^{71} \mathrm{On}$ the other hand, $a b$ initio theory ${ }^{77,78}$ predicts interstial levels $0.9 \mathrm{eV}$ above the valence band. Comparison of our numerical calculations with experimental data ${ }^{33}$ could thus suggest that the localized states near the conduction band arise from interstitials rather than from antisites. However, the discrepancy of theoretical and experimental fits near $1 \mathrm{eV}$ could also suggest a scenario with a broader impurity band or a merging of the conduction band with the impurity band. Another possible scenario, suggested by photoluminescence and optical absorption experiments on GaN:Mn,Mg (Refs. 81, 83 , and 84 ) and $\mathrm{GaN}: \mathrm{Fe},{ }^{82}$ is that part of the upturn in the spectra in the optical range is due to intra- $d$-shell transitions. We believe the resolution of this question can be determined 


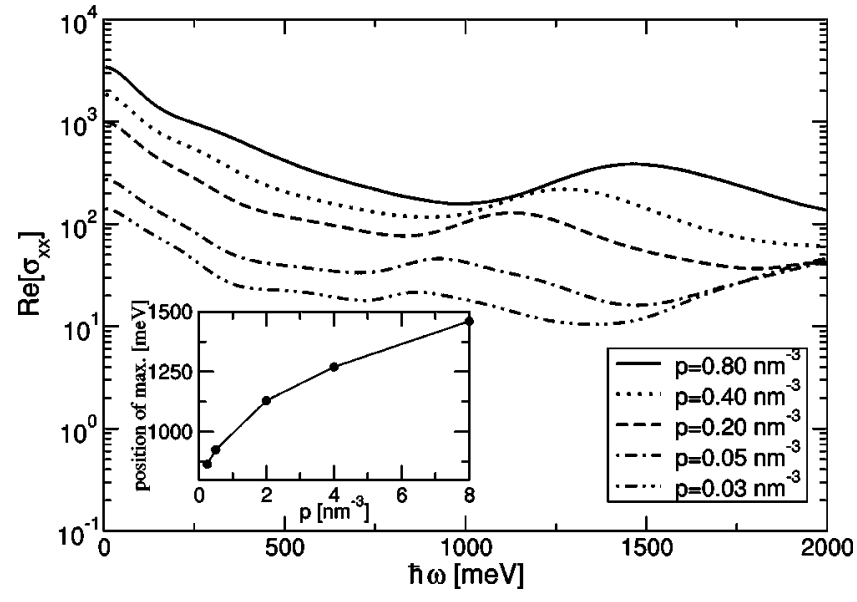

FIG. 6. Real part of the diagonal conductivity as a function of frequency for $\mathrm{Ga}_{0.96} \mathrm{Mn}_{0.04} \mathrm{As}$ and various hole concentrations. In the inset the position of the maximum corresponding to transitions between valence and defect band is plotted vs carrier concentration. Here, the dispersionless defect energy level $E_{g}^{\prime}$ is $0.75 \mathrm{eV}$ above the valence band. The dipole energy between the defect level and the valence band is $E_{\text {an }}=2.2 \mathrm{eV}$.

by further experiments on optimally annealed samples, for which this theory is designed, in particular on metallic samples with dc conductivities above a few hundred $\Omega^{-1} \mathrm{~cm}^{-1}$.

In Fig. 6 the total real part of the diagonal conductivity is plotted as a function of frequency for different concentrations of holes. We assume that the localized states form a flat band $0.75 \mathrm{eV}$ above the valence band.

Thus on the basis of experimental data $^{71}$ for undoped GaAs they might correspond to antisite states. In the lowfrequency range the conductivity is similar to the one obtained from the six- and eight-band models, as expected. However, the additional transition between the valence band and the antisite band appears around $800 \mathrm{meV}$ for the strongly compensated sample $\left(p=0.025 \mathrm{~nm}^{-3}\right.$ for a Mn concentration of $x=4 \%$ ). The peak from this transition shifts in the direction of higher energy with increasing carrier concentration. In the inset we plot the position of the maximum due to the transition between valence and defect band as a function of frequency. This dependence can be useful for rough estimates of carrier concentrations on the basis of optical and infrared conductivities.

The MCD signal should change with the thickness of the (Ga,Mn)As film (geometry effect) as well as with the number of allowed transitions (band-structure effect). In the thin-film geometry the condition $d / \lambda<1$ should be fulfilled, leading to $d<200 \mathrm{~nm}$ for visible light. However, the (Ga,Mn)As films measured so far have a thickness of 100-500 nm. The results presented below consider the structure air/(Ga, Mn)As/GaAs and take multiple reflections into account.

In Fig. 7 we compare the theoretical results for the MCD signal for eight- and ten-band models in the thin-film limit and for a film with a larger thickness of $d=500 \mathrm{~nm}$. In the case of $d=500 \mathrm{~nm}$ film the absorption for the right and left polarized light is defined as

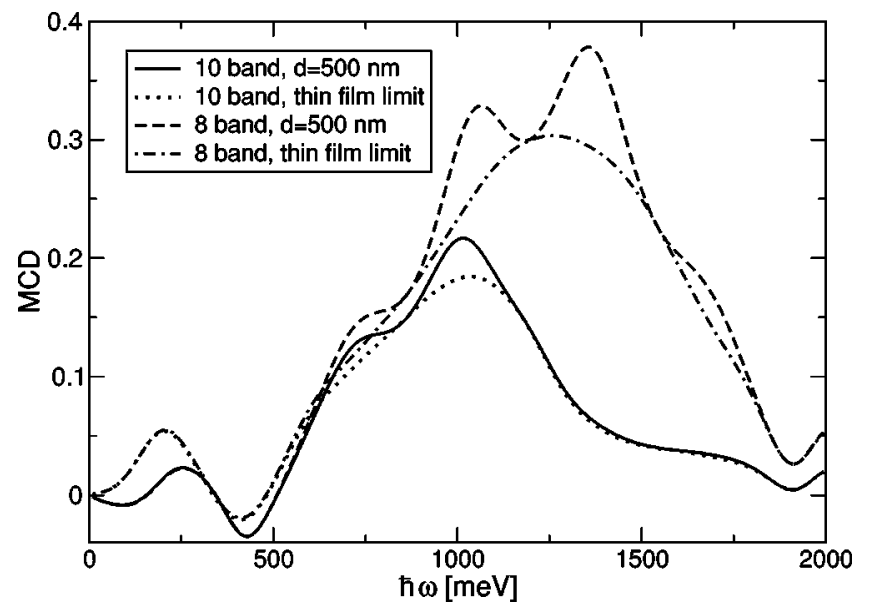

FIG. 7. Comparison of the theoretical MCD signal for eight- and ten-band models for a $\mathrm{Ga}_{0.95} \mathrm{Mn}_{0.05}$ As epilayer on $\mathrm{GaAs}$ substrate. The dotted and dashed-dotted curves correspond to a thin-film geometry. The solid and long-dashed curves refer to a film of thickness $d=500 \mathrm{~nm}$.

$\alpha^{ \pm}=\left(1-\left|t_{\mathrm{air} / \mathrm{GaMnAs} / \mathrm{GaAs}}^{ \pm} / t_{\mathrm{air} / \mathrm{GaAs}}^{ \pm}\right|^{2}\right) / d$, where $t_{\mathrm{air} / \mathrm{GaMnAs} / \mathrm{GaAs}}^{ \pm}$ are the transmissions for the right and left polarized light in all structure air/(Ga, Mn)As/GaAs and $t_{\text {air/GaAs }}^{ \pm}$are the transmissions for the right and left polarized light between air and GaAs. Note that the interference peaks appear for maximal values of $e^{i d / \lambda}$ and are superimposed on the MCD signal for $d=500 \mathrm{~nm}$. The calculations for different film thicknesses show that the interference peaks change positions, as expected. However, the common effect is an increase of the MCD signal around the optical transitions. The difference of theoretical predictions for eight- and ten-band models is quite pronounced. The maximum at low photon energies corresponds to transitions between valence subbands, as discussed above, and is seen for both eight- and ten-band models. However, for the eight-band model the amplitude of MCD at high photon energies is almost twice that for the ten-band model. The main MCD signal is concentrated around the valence-conduction-band transition for the eightband model while it is around the valence-defect-band transition for the ten-band calculation. These results agree qualitatively with the experiments. The MCD spectra of LT-GaAs (Ref. 72) show that the MCD signal for antisites lies around $1 \mathrm{eV}$ in agreement with the ten-band model. The theoretical MCD spectrum has a much larger amplitude for the eightband model and is shifted to higher energies in agreement with experiments. ${ }^{41}$

\section{SUMMARY}

We have presented a detailed analysis of the optical and infrared conductivity as well as of magneto-optical effects in the infrared to optical range. The mean-field/VCA approach using the eight- and ten-band KL model coupled with $S$ $=5 / 2 \mathrm{Mn}$ spins by a local exchange interaction has been applied. We have shown that the ten-band KL model with additional disspersionless bands simulating phenomenologically the upper-mid-gap states is successful in explaining 
qualitatively the recent infrared and optical conductivity measurements by Singley et al. ${ }^{33}$ Also, we have predicted strong novel signatures in the magneto-optical effects in the mid-infrared range which are due to the strong spin-orbit coupling present in these materials which allow intervalence band transitions in these highly doped samples.

We hope that the predicted dependence of the optical conductivity as well as the MCD signal and the Kerr effect on the concentrations of carriers will motivate new experimental efforts to understand the fascinating magneto-optical properties of diluted magnetic semiconductors. In particular, this dependence can provide a test of the validity of the effective
Hamiltonian model in describing the optical properties of these materials.

\section{ACKNOWLEDGMENTS}

We would like to thank W. A. Atkinson, A. H. MacDonald, H. Ohno, K. S. Burch, D. N. Basov, and J. Mašek, for useful discussions. The work by T.D. was partly supported by FENIKS Project No. EC:G5RD-CT-2001-00535. This work was further supported by the Welch Foundation, the DOE under Grant No. DE-FG03-02ER45958, and the Grant Agency of the Czech Republic under Grant No. 202/ 02/0912.
${ }^{1}$ H. Ohno, Science 281, 951 (1998).

${ }^{2}$ H. Ohno, J. Magn. Magn. Mater. 200, 110 (1999).

${ }^{3}$ T. Dietl, Acta Phys. Pol. A 100, 139 (2001).

${ }^{4}$ T. Dietl and H. Ohno, MRS Bull. 28, 714 (2003).

${ }^{5}$ H. Ohno, H. Munekata, T. Penney, S. von Molnár, and L. L. Chang, Phys. Rev. Lett. 68, 2664 (1992).

${ }^{6}$ H. Ohno, A. Shen, F. Matsukura, A. Oiwa, A. Endo, S. Katsumoto, and Y. Iye, Appl. Phys. Lett. 69, 363 (1996).

${ }^{7}$ T. Hayashi, Y. Hashimoto, S. Katsumoto, and Y. Iye, Appl. Phys. Lett. 78, 1691 (2001).

${ }^{8}$ K. Edmonds, K. Wang, R. Campion, A. Neumann, N. Farley, B. Gallagher, and C. Foxon, Appl. Phys. Lett. 81, 4991 (2002).

${ }^{9}$ K. C. Ku, S. J. Potashnik, R. F. Wang, S. H. Chun, P. Schiffer, N. Samarth, M. J. Seong, A. Mascarenhas, E. Johnston-Halperin, R. C. Myers, A. C. Gossard, and D. D. Awschalom, Appl. Phys. Lett. 82, 2302 (2003).

${ }^{10}$ K. M. Yu, W. Walukiewicz, T. Wojtowicz, I. Kuryliszyn, X. Liu, Y. Sasaki, and J. K. Furdyna, Phys. Rev. B 65, 201303(R) (2002).

${ }^{11}$ K. W. Edmonds, P. Bogusławski, K. Y. Wang, R. P. Campion, S. N. Novikov, N. R. S. Farley, B. L. Gallagher, C. T. Foxon, M. Sawicki, T. Dietl, M. B. Nardelli, and J. Bernholc, Phys. Rev. Lett. 92, 037201 (2004).

${ }^{12}$ I. Kuryliszyn-Kudelska, T. Wojtowicz, X. Liu, J. K. Furdyna, W. Dobrowolski, J. Z. Domagala, E. Lusakowska, M. Goiran, E. Haanappel, and O. Portugall, cond-mat/0304622 (2003).

${ }^{13}$ J. Schneider, U. Kaufmann, W. Wilkening, M. Baeumler, and F. Köhl, Phys. Rev. Lett. 59, 240 (1987).

${ }^{14}$ M. Linnarsson, E. Janzén, B. Monemar, M. Kleverman, and A. Thilderkvist, Phys. Rev. B 55, 6938 (1997).

${ }^{15}$ J. Szczytko, A. Twardowski, M. Palczewska, R. Jablonski, J. Furdyna, and H. Munekata, Phys. Rev. B 63, 085315 (2001).

${ }^{16}$ J. Szczytko, A. Twardowski, K. Swiatek, M. Palczewska, T. H. M. Tanaka, and K. Ando, Phys. Rev. B 60, 8304 (1999).

${ }^{17}$ O. M. Fedorych, E. M. Hankiewicz, Z. Wilamowski, and J. Sadowski, Phys. Rev. B 66, 045201 (2002).

${ }^{18}$ T. Dietl, H. Ohno, F. Matsukura, J. Cibert, and D. Ferrand, Science 287, 1019 (2000).

${ }^{19}$ T. Dietl, H. Ohno, and F. Matsukura, Phys. Rev. B 63, 195205 (2001)

${ }^{20}$ J. König, J. Schliemann, T. Jungwirth, and A. H. MacDonald, Electronic Structure and Magnetism of Complex Materials
(Springer Verlag, Berlin, 2002).

${ }^{21}$ T. Jungwirth, J. König, J. Sinova, J. Kučera, and A. H. MacDonald, Phys. Rev. B 66, 012402 (2002).

${ }^{22}$ T. Jungwirth, J. Sinova, K. Wang, K. W. Edmonds, R. Campion, B. Gallagher, C. Foxon, Q. Niu, and A. MacDonald, Appl. Phys. Lett. 83, 320 (2003).

${ }^{23}$ T. Jungwirth, Q. Niu, and A. MacDonald, Phys. Rev. Lett. 88, 207208 (2002).

${ }^{24}$ T. Jungwirth, M. Abolfath, J. Sinova, J. Kučera, and A. MacDonald, Appl. Phys. Lett. 81, 4029 (2002).

${ }^{25}$ M. Abolfath, T. Jungwirth, J. Brum, and A. MacDonald, Phys. Rev. B 63, 054418 (2001).

${ }^{26}$ J. König, T. Jungwirth, and A. H. MacDonald, Phys. Rev. B 64, 184423 (2001).

${ }^{27}$ T. Dietl, J. König, and A. H. MacDonald, Phys. Rev. B 64, 241201 (2001).

${ }^{28}$ J. Sinova, T. Jungwirth, Y. S. X. Liu, J. Furdyna, W. A. Atkinson, and A. MacDonald, cond-mat/0308386 (2003).

${ }^{29}$ J. Sinova, T. Jungwirth, S.-R. E. Yang, J. Kučera, and A. H. MacDonald, Phys. Rev. B 66, 041202 (2002).

${ }^{30}$ J. Sinova, T. Jungwirth, J. Kučera, and A. H. MacDonald, Phys. Rev. B 67, 235203 (2003).

${ }^{31}$ C. Timm, J. Phys.: Condens. Matter 15, R1865 (2003).

${ }^{32}$ E. J. Singley, R. Kawakami, D. D. Awschalom, and D. N. Basov, Phys. Rev. Lett. 89, 097203 (2002).

${ }^{33}$ E. J. Singley, K. S. Burch, R. Kawakami, J. Stephens, D. D. Awschalom, and D. N. Basov, Phys. Rev. B 68, 165204 (2003).

${ }^{34}$ R. A. Chapman and W. G. Hutchinson, Phys. Rev. Lett. 18, 443 (1967).

${ }^{35}$ K. Hirakawa, S. Katsumoto, T. Hayashi, Y. Hashimoto, and Y. Iye, Phys. Rev. B 65, 193312 (2002).

${ }^{36}$ K. Hirakawa, A. Oiwa, and H. Munekata, Physica E (Amsterdam) 10, 215 (2001).

${ }^{37}$ Y. Nagai, T. Kunimoto, K. Nagasaka, H. Nojiri, M. Motokawa, G. Matsukura, T. Dietl, and H. Ohno, Jpn. J. Appl. Phys., Part 1 40, 6231 (2001).

${ }^{38}$ J. Szczytko, W. Mac, A. Twardowski, F. Matsukura, and H. Ohno, Phys. Rev. B 59, 12935 (1999).

${ }^{39}$ J. Szczytko, A. Stachow, W. Mac, A. Twardowski, P. Belca, and J. Tworzydlo, Acta Phys. Pol. A 90, 951 (1996).

${ }^{40}$ K. Ando, T. Hayashi, M. Tanaka, and A. Twardowski, J. Appl. Phys. 83, 6548 (1998). 
${ }^{41}$ B. Beschoten, P. A. Crowell, I. Malajovich, D. D. Awschalom, F. Matskura, A. Shen, and H. Ohno, Phys. Rev. Lett. 83, 3073 (1999).

${ }^{42}$ V. F. Sapega, T. Ruf, and M. Cardona, Phys. Status Solidi B 226, 339 (2001).

${ }^{43}$ M. J. Seong, S. H. Chun, H. M. Cheong, N. Samarth, and A. Mascarenhas, Phys. Rev. B 66, 033202 (2002).

${ }^{44}$ W. Limmer, M. Glunk, S. Mascheck, A. Koeder, D. Klarer, W. Schoch, K. Thonke, R. Sauer, and A. Waag, Phys. Rev. B 66, 205209 (2002).

${ }^{45}$ J. Okabayashi, A. Kimura, O. Rader, T. Mizokawa, A. Fujimori, T. Hayashi, and M. Tanaka, Phys. Rev. B 58, R4211 (1998).

${ }^{46}$ J. Okabayashi, A. Kimura, T. Mizokawa, A. Fujimori, T. Hayashi, and M. Tanaka, Phys. Rev. B 59, R2486 (1999).

${ }^{47}$ J. Okabayashi, T. Mizokawa, D. D. Sarma, A. Fujimori, T. Slupinski, A. Oiwa, and H. Munekata, Phys. Rev. B 65, 161203 (2002).

${ }^{48}$ J. Okabayashi, A. Kimura, O. Rader, T. Mizokawa, A. Fujimori, T. Hayashi, and M. Tanaka, Phys. Rev. B 64, 125304 (2001).

${ }^{49}$ H. Åsklund, L. Ilver, J. Kanski, J. Sadowski, and R. Mathieu, Phys. Rev. B 66, 115319 (2002).

${ }^{50}$ G. A. Khodaparast, J. Kono, Y. H. Matsuda, S. Ikeda, N. Miura, T. Slupinski, A. Oiwa, H. Munekata, Y. Sun, F. V. Kyrychenko et al., cond-mat/0307087 (2003).

${ }^{51}$ G. D. Sanders, Y. Sun, F. V. Kyrychenko, C. J. Stanton, G. A. Khodaparast, M. A. Zudov, J. Kono, Y. H. Matsuda, N. Miura, and H. Munekata, Phys. Rev. B 68, 165205 (2003).

${ }^{52}$ Y. Mitsumori, A. Oiwa, T. Slupinski, H. Maruki, Y. Kashimura, F. Minami, and H. Munekata, cond-mat/0307268 (2003).

${ }^{53}$ K. S. Burch, J. Stephens, R. K. Kawakami, D. D. Awschalom, and D. N. Basov, Phys. Rev. B 70, 205208 (2004).

${ }^{54}$ S.-R. E. Yang, J. Sinova, T. Jungwirth, Y. Shim, and A. MacDonald, Phys. Rev. B 67, 045205 (2003).

${ }^{55}$ W. Kohn and J. Luttinger, Phys. Rev. 108, 590 (1957).

${ }^{56}$ E. H. Hwang, A. J. Millis, and S. D. Sarma, Phys. Rev. B 65, 233206 (2002).

${ }^{57}$ G. Alvarez and E. Dagotto, Phys. Rev. B 68, 045202 (2003).

${ }^{58}$ T. Omiya, F. Matsukura, T. Dietl, Y. Ohno, T. Sakon, M. Motokawa, and H. Ohno, Physica E (Amsterdam) 7, 976 (2000).

${ }^{59}$ J. Szczytko, W. Bardyszewski, and A. Twardowski, Phys. Rev. B 64, 075306 (2001).

${ }^{60}$ J. Schliemann, Phys. Rev. B 67, 045202 (2003).

${ }^{61}$ G. Zaránd and B. Jankó, Phys. Rev. Lett. 89, 047201 (2002).
${ }^{62}$ L. Brey and G. Gomez-Santos, Phys. Rev. B 68, 115206 (2003).

${ }^{63}$ J. Blinowski and P. Kacman, Phys. Rev. B 67, 121204 (2003).

${ }^{64}$ R. Mathieu, B. S. Sørensen, J. Sadowski, J. Kanski, P. Svedlindh, and P. E. Lindelof, cond-mat/0208411 (2002).

${ }^{65}$ T. E. Ostromek, Phys. Rev. B 54, 14467 (1996).

${ }^{66}$ J. H. C. Casey and F. F. Stern, J. Appl. Phys. 47, 631 (1976).

${ }^{67}$ P. R. Rimbey and G. D. Mahan, Phys. Rev. B 10, 3419 (1974).

${ }^{68}$ Here we use the convention of defining the right circular polarized light as the cyclotron active $\left(\sigma^{+}\right)$light following the convention used in previous experiments. Other conventions, such as the screw or angular momentum convention, are also used by ohter physics and engineering communities.

${ }^{69} \mathrm{~K}$. Ando, in Magneto-Optics, edited by S. Sugano and N. Kojima (Springer-Verlag, Berlin, 2000), p. 211.

${ }^{70}$ M. Kaneko, in Magneto-Optics, edited by S. Sugano and N. Kojima (Springer-Verlag, Berlin, 2000), p. 271.

${ }^{71}$ E. R. Weber, H. Ennen, U. Kaufmann, J. Windscheif, J. Schneider, and T. Wosinski, J. Appl. Phys. 53, 6140 (1982).

${ }^{72}$ B. K. Meyer, J.-M. Spaeth, and M. Scheffler, Phys. Rev. Lett. 52, 851 (1984).

${ }^{73}$ U. Kaufmann and J. Windscheif, Phys. Rev. B 38, 10060 (1988).

${ }^{74}$ A. Prasad, P. Stallinga, X. Liu, and E. R. Weber, Phys. Rev. B 57, R4214 (1998).

${ }^{75}$ M. Kaminska, M. Skowronski, and W. Kuszko, Phys. Rev. Lett. 55, 2204 (1985).

${ }^{76}$ J. C. Bourgoin, H. J. von Bardeleben, and D. Stiévenard, J. Appl. Phys. 64, 65 (1988).

${ }^{77}$ S. C. Erwin and A. G. Petukhov, Phys. Rev. Lett. 89, 227201 (2002).

${ }^{78}$ J. Kudrnovský, I. Turek, V. Drchal, F. Máca, J. Masek, P. Weinberger, and P. Bruno, J. Supercond. 16, 119 (2003).

${ }^{79}$ C. Timm, F. Schäfer, and F. von Oppen, Phys. Rev. Lett. 89, 137201 (2002).

${ }^{80}$ C. Timm and F. von Oppen, J. Supercond. 16, 23 (2003).

${ }^{81}$ A. Wolos, M. Palczewska, M. Zajac, J. Gosk, M. Kaminska, A. Twardowski, M. Bockowski, I. Grzegory, and S. Porowski (unpublished).

${ }^{82}$ R. Heitz, P. Maxim, L. Eckey, P. Thurian, A. Hoffmann, I. Broser, K. Pressel, and B. K. Meyer, Phys. Rev. B 55, 4382 (1997).

${ }^{83}$ R. Y. Korotkov, J. M. Gregie, and B. W. Wessels, Physica B 308, 33 (2001).

${ }^{84}$ T. Graf, M. Gjukic, M. S. Brandt, M. Stutzmann, and O. Ambacher, Appl. Phys. Lett. 81, 5159 (2002). 